\title{
TEXTSORTEN IN DER INTERKULTURELLEN KOMMUNIKATION - EIN PROBLEMAUFRIß
}

1. Jede Textklassifikation folgt dem Ziel, die unendliche Vielfalt realer Texte in der kommunikativen Praxis auf überschaubare Grundtypen zu reduzieren. Dieses Ziel ist von wissenschaftlichem, aber auch von pädagogischem Interesse; denn das, was wir genau kennen bzw. zu kennen glauben, läßt sich in einen übergreifenden Zusammenhang einordnen, es läßt sich beschreiben und auch lehren. Die Textsortenforschung steht keineswegs am Anfang, șo daß sie durchaus Ergebnisse vorweisen kann, die bereits Lehrgegenstand in den Schulen und Hochschulen sind. Die Deutsch-Schulbücher des Cornelsen-Verlags für Schüler an deutschen Gymnasien verwenden wie selbstverständlich den Begriff der Textsorte und beziehen ihn auf nichtliterarische Texte, z.B. auf "Glosse", "Reportage", "Kommentar". Offenbar ist der textlinguistische Terminus "Textsorte" bereits zum populärwissenschaftlichen Fachbegriff geworden und gehört heute auch zur Schulterminologie.

Der Textsortenbegriff ist ein Produkt der kommunikativen Sprachbetrachtung, das Textsortenwissen beruht auf überlieferter Alltagserfahrung aus unzähligen Akten sprachlich-kommunikativer Tätigkeit, das heißt, es ist im Alltagsbewußtsein verankert. Das Problem, das wir Linguisten uns anschicken zu lösen, besteht in seinem Kern darin, es aus dem Alltagsbewußtsein in wissenschaftliches Bewußtsein umzuwandeln, oder noch genauer gesagt, einen wissenschaftlichen Textsortenbegriff zu entwickeln, der die bekannten Schwierigkeiten bei der Handhabung des alltäglichen und individuell gespeicherten Textsortenmusters überwinden hilft (vgl. dazu E. Gülich 1986, 15 ff.).

Die Fortschritte in der Textsortenforschung haben nicht nur neue Zielrichtungen aufgezeigt, sie haben auch das Problemfeld erweitert. Das spricht für ein nach wie vor aktuelles Forschungsinteresse. Soweit ich die Literatur übersehe - und das ist zur Gänze wohl kaum noch möglich - richten sich die Forschungen hauptsächlich auf folgende Ziele:

(a) Textsortenforschung als Wegbereitung für die Ausarbeitung einer theoretisch befriedigenden Texttypologie;

(b) Erkundung des Textsortenpotentials einer kulturell homogenen Kommunikationsgemeinschaft

(c) empirische Untersuchungen zu ausgewählten, kulturell homogenen Textsorten und Realisierungsvarianten 
(d) Forschung im Hinblick auf notwendiges Textsortenwissen für die kulturelle und interkulturelle Kommunikation; einzelsprachliche und übereinzelsprachliche Realisierungen

(e) Textsortenwissen in einer Verstehensstrategie

(f) Textsortenbeschreibung für alltägliche Lebensbereiche und praktische Bedürfnisse, z.B. berufliche Tätigkeit, Geschäftskorrespondenz, soziale Kontakte. Die Ziele reichen weiter als hier festgestellt werden kann, sie sind zumeist noch spezieller und differenzierter.

Die Auffassungen darüber, was eine Textsorte sei, gehen noch immer weit auseinander. $\mathrm{Zu}$ Beginn der Textsortenforschung Ende der 60er, Anfang der 70er Jahre wurde nahezu alles mit dem Textsortenbegriff belegt, was sich durch Abstraktion realer Textvorkommen zu Klassen vereinen ließ. Auf dem 1972 in Deutschland durchgeführten Kolloquium zu "Differenzierungskriterien für Textsorten aus der Sicht der Linguistik und einzelner Textwissenschaften" kamen die Veranstalter zu folgendem Resümee:

"... zum Teil wird der Begriff der 'Textsorte' sehr eng gefaßt, wie etwa von Barbara Sandig, die u.a. 'Kochrezept', 'Arztrezept' und 'Gebrauchsanweisung' als verschiedene Textsorten ansieht. Von anderen wird der Begriff sehr weit gefaßt, etwa von Siegfried J. Schmidt, der fiktionale Texte als eine Textsorte behandelt, von Wolfgang Dreßler, der eine Textsorte 'Übersetzung' etabliert, von Werner Kummer, der eine Textsorte 'Argumentation' postuliert, und von der Konstanzer Gruppe, die ganz bewußt mit dem sehr vagen Oberbegriff 'Narrative Strukturen' arbeitet" (E. Gülich/ W. Raible 1972, 2).

Zieht man zum Vergleich die aktuelle Diskussion heran, dann hat man zunächst den Eindruck, daß sich in der Textsortendiskussion der 80er Jahre kaum etwas bewegt hat. E. Oksaar kommt etwa zu dem gleichen Resultat, wenn sie registriert: "Die sogenannte pragmatische Wende, so vielversprechend wie sie auch gewesen sein mag, scheint jedoch in den 80er Jahren allgemein nicht wesentlich an Kreativität gewonnen zu haben" $(1988,14)$. Auf der Konferenz "Textsorten/Textmuster" 1990 in Leipzig wird teils Bilanz gezogen, teils aber auch nach weiterführenden Erklärungen zwischen Theorie und Praxis gesucht. Vor allem Wolfgang Heinemann resümierte seine Problemsicht. Dabei stellte er - hier nur thesenhaft wiedergegeben - folgende Probleme heraus:

(1) Der Versuch einer typologischen Textsortenbestimmung in Termen distinktiver Merkmale, wie sie zum Beispiel von Barbara Sandig 1972 publiziert wurde (1972, 122), war zwar der am besten ausgearbeitete Vorschlag für eine kommunikativ orientierte Typologie, aber er erwies sich in dem Moment als unzureichend, als man versuchte, auf dieser Basis ein strigentes und in sich geschlossenes System von Textsorten zusammenzustellen. Es erwies sich, daß es vielfach Überlappungen von den in der kommunikativen Praxis erfahrenen Textsorten gibt, daß sie in Implikationsbeziehungen zueinander stehen, daß also letztlich dieses hypothetische System nicht ausbaufähig ist.

Zum besseren Verständnis möchte ich verdeutlichen, wovon hier die Rede ist: Ein Gesetzestext hat die Merkmale *monologisch, *festgelegtes Thema, *bestimmte Form 
des Textanfangs, *bestimmte Form des Textendes, *enthält Nichtsprachliches (z.B. Zeichen für §). Ein Arztrezept unterscheidet sich davon durch die Merkmale *ökonomische Formen und *weitgehend festgelegter Textaufbau (ebenda, 122). In den anderen Merkmalen stimmen beide Textsorten überein.

(2) Die Vorstellungen für eine wissenschaftliche Klassifikation waren an mathematische Kriterien gebunden worden (beispielsweise von $\mathrm{H}$. Isenberg): an Vollständigkeit des Systems, Homogenität, Monotypie, Striktheit. Dieses deduktiv hergeleitete System forderte starre Rahmenstrukturen für den Textaufbau, für die sprachliche Typik, für die kommunikativen Bedingungen. Von den Kommunizierenden mußte ein sehr genaues Textsortenwissen erwartet werden, wenn sie die Anforderungen an eine Textsorte erfüllen wollten.Dieses theoretische Konstrukt mußte schon deshalb aufgegeben werden, weil Stilentscheidungen ebenso variieren wie die kommunikativen Bedingungen, unter denen sich sprachliches Handeln vollzieht, - und natürlich auch variieren müssen. Für vollständig genormte oder teilweise genormte Gebrauchsformen wie tabellarischer Lebenslauf, Unfallanzeige per Formblatt, Steuererklärung, computergenormtes amtliches Schreiben können die oben genannten Kriterien zugrunde gelegt werden. Nur dann wären Textsorten als Gruppe von Textklassen zu bestimmen, die institutionell genormt sind und als Gebrauchsformen vorwiegend in der behördlichen Kommunikation Anwendung finden. Seitens der ethnomethodologischen Forschung wurden die starren Rahmenbedingungen für Textsorten in Frage gestellt. Man denke nur an Texte der spontanen, vornehmlich mündlichen Kommunikation. Das Problem besteht nach wie vor darin, daß Versuche der Textsortendifferenzierung erschwert werden durch die mehr oder minder ausgeprägte Variabilität in der Textgestaltung (in Sprache, Textaufbau, Textintention, Textthema).

Offensichtlich aber ist mit dem Begriff des Prototypischen ein Lösungsansatz gefunden worden, der überlieferte relativ festgewordene psychische Orientierungsmuster, gewissermaßen Schemata, impliziert. Diese Schemata werden den situativen Bedingungen angepaßt und müssen deshalb variabel sein. Als Beispiel kann die Textsorte "Einladung" angeführt werden. Sie variiert in den Realisierungsformen "offizielle Einladung", "inoffizielle mündliche Einladung", "inoffizielle schriftliche Einladung". Dazu können Subformen genannt werden: "persönliche Einladung", "allgemeine Einladung". Die Subformen können durch die Angabe des Grundes weiter spezifiziert sein: "Partyeinladung", "Konferenzeinladung", "Einladung zum Empfang", "Einladung zu einer Ausstellung", Einladung zum Abendessen, zum Kaffee, zum Geburtstag. Alle genannten Realisierungsformen variieren mehr oder minder in der sprachlichen Struktur, sie werden unter verschiedenen situativen Bedingungen realisiert, also unter verschiedenen sozialen Bedingungen, unter den Bedingungen verschiedener Kommunikationsbereiche, beispielsweise Alltag, Wissenschaft, Diplomatie. Ebenso variieren auch die Intentionen, mit denen die Einladungen erfolgt sind. Dennoch läßt sich eine Invarianz feststellen, die es dem Rezipienten ermöglicht, die Textsorte zu erkennen: Es ist das Substantiv "Einladung" bzw. das Verb "einladen", und es ist die Angabe des Grundes. 
Für die Einladung dominiert offensichtlich die Varianz, für andere Textsorten kann es die Invarianz sein, z.B. für den Geschäftsbrief, für das Kochrezept, für alle formalisierten Textsorten. Das Beispiel der Einladung provoziert Fragen, die wiederum Probleme anzeigen:

(a) Kann eine Klasse von Textvorkommen mit sehr geringer Invarianz und folglich hohem Verallgemeinerungsgrad als Textsorte wissenschaftlich beschrieben werden? Handelt es sich nicht vielmehr um Textty pen, wenn man den Typ als theoriebezogene Kategorie begreift, der über globale Invarianten definiert wird? (Ich komme darauf noch zurück) - Auch B. Sandig läßt das Invarianzkriterium weitgehend außer Acht, wenn sie den Brief als eine Textsorte undifferenziert neben Wetterbericht und Kochrezept stellt. Konsequenterweise müßten dann allgemeinere Formen wie Rezept und Bericht als Textsorten aufgefaßt werden.

(b) Die Beispiele lenken uns auf eine generelle Fragestellung: Welches Textvorkommen in der sprachlichen Kommunikation soll eigentlich mit dem Begriff "Textsorte" belegt und beschrieben werden? Da sich Forschung nicht im Selbstzweck erschöpft, sondern ihre Ergebnisse von praktischem und theoretischem Wert sind (bzw. sein sollten), da die Textsorte - nach allem, was die Diskussion bisher erbracht hat - im Alltagsbewußtsein verankert ist und deshalb in der Hierarchie der Textklassen wohl die untere Ebene belegt, kann sie meines Erachtens nur als eine sehr spezielle Bezeichnung für eine Klasse von Texten verstanden werden. Der Begriff von der Textsorte ist - wie andere Begriffe auch - ein mehr oder weniger gesellschaftlich durchschnittliches Abbild im menschlichen Bewußtsein, das als Wissensmuster gespeichert ist und in kommunikativen Akten als Orientierungsrahmen dient. Wie das Wissensmuster beschaffen ist, hängt letztlich von den konkreten Anforderungen ab, die an den Sprecher/Schreiber in seiner Tätigkeit an ihn gestellt sind. Wer von Berufs wegen Protokolle zu schreiben hat, sei es nun ein stenographisches Protokoll, ein Verlaufsprotokoll oder ein Ergebnisprotokoll, hat dafür auch Wissensmuster erworben, die durch verbindliche Merkmale, durch Invarianten, gekennzeichnet sind. Die Linguisten sehen sich - unter anderem natürlich - vor die Aufgabe gestellt, solche Wissensmuster zu beschreiben und allgemein zugängig zu machen. Ich verweise nur auf die Fachtextsorten-Forschung, speziell auf Textsorten in der Wirtschaft und in der Diplomatie.

Die allgemeinbildende Schule hat zweifellos sehr viel zur Ausbildung der Textsortenmuster beigetragen, ich erinnere nur an Muster für den "tabellarischen Lebenslauf", für "Protokoll", "Diskussionsbeitrag". Ich erinnere auch an Textsorten der Massenmedien wie "Pressenachricht", "Leserbrief", "Feuilleton". Doch die allgemeinbildende Schule muß sich auf die Vermittlung allgemeinen Wissens beschränken, das später ohnehin durch weiterführende Schulen für die konkreten Anforderungen der Berufspraxis ausgebaut wird. Es genügt offenbar zunächst zu wissen, wie ein Bericht sprachlich beschaffen und gegliedert ist. Im Schulbuch des Cornelsenverlags für das 7 . Schuljahr, 1. Auflage 1991, wird "Bericht" wie folgt definiert: 
"Ein Bericht ist ein Gebrauchstext, der in erster Linie der sachlichen Information über einen Vorgang, ein Ereignis u.ä. dient. Der Berichtende sollte sich deshalb persönlicher Wertungen und Beurteilungen enthalten und sich auf die klare und möglichst genaue Darstellung des Geschehens oder des Sachverhalts beschränken." (Lesen, Darstellen, Begreifen. - Lese- und Arbeitsbuch für den Sprachunterricht, Ausgabe A, 7. Schuljahr, Frankfurt/Main 1991, Seite 357)

Die Definition umreißt einen allgemeinen Wissensrahmen, ein Grundmuster, mit den Merkmalen "sachlich", der "Information dienend", "relativ frei von persönlichen Wertungen", "möglichst genaue Darstellung", "authentisch". Es werden Anforderungen an Proposition (Sachverhaltsdarstellung), Intention und Sprache gestellt, die gewissermaßen auf den Kern des Wissensrahmens bezogen sind. Selbst meine ich, daß diese Orientierung für viele Lebenssituationen ausreicht. Eine Textsorte ist mit diesen Merkmalen aber noch nicht umrissen, denn für die Praxis wird eine Differenzierung erwartet, aus der textsortenspezifische Merkmale hervorgehen, z.B. für ein Verlaufsprotokoll, einen Untersuchungsbericht, einen Fallbericht; eine solche Differenzierung ist zweckmäßiger.

Berichte sind ergebnisorientiert und zumeist institutionsgebunden (vgl. B. Sandig 1986, 184). Das erklärt auch die zahlreichen Textsorten, die auf dem Grundmuster zum "Bericht" basieren. Wörterbücher, Lehrmaterialen und Lehrbücher, auch wissenschaftliche Abhandlungen weisen als berichtende Textsorten aus: Lebenslauf mit seinen Realisierungsformen "ausführlicher und tabellarischer Lebenslauf", Protokoll mit seinen Realisierungsformen "Verlaufs- und Ergebnisprotokoll", Untersuchungsbericht mit zahlreichen Realisierungsformen, die nach ihren Berichterstattern benannt sind, z.B. Untersuchungsbericht der Arbeitsschutzkommission, der Ärztekammer, des parlamentarischen Ausschusses, der Ärztekommission. Auch eine sogenannte "Befundung" nach medizinischen Untersuchungen kann der Textsorte "Untersuchungsbericht" entsprechen. Nebengeordnete Textsorten sind Anamnese (Krankengeschichte) und Krankenbericht. Variantenreich ist auch der "Fallbericht", mit dem ein Vorkommnis aufgezeichnet ist. Eine seiner Formen ist beispielsweise der "Unfallbericht". Nicht betont sachlich ist der "Sportbericht". Im Unterschied zur "Sportnachricht" (eine Mitteilung über ein Sportereignis in den Medien) ist der Sportbericht eher aktionsgeladen, er ist nicht frei von Wertungen und Emotionen.

Medienberichte weichen vom Grundmuster des Texttyps "Bericht" ab. Das ist gewiß keine Opposition gegen Konventionen, sondern die "Abweichung" beruht auf Erwartungen an die Medienkommunikation. Damit wird ein textexternes Merkmal ins Spiel gebracht, das man als "situatives Merkmal" bezeichnen kann; situativ mit Bezug auf den Kommunikationsbereich, in dem kommuniziert wird. Verallgemeinernd läßt sich als Hypothese formulieren, daß die erwartete musterhafte Grundstruktur ein differenzierendes Merkmal einschließt, das auf den Kommunikationsbereich verweist. Danach wäre zu differenzieren zwischen institutionsgebundenem (das heißt offiziellem) Bericht, Medienbericht, Alltagsbericht. Die in Lehrbüchern verbreitete Auffassung vom 
Bericht bezieht sich eindeutig auf den "offiziellen" Bericht und das ihm entsprechende Grundmuster. Auch im Kommunikationsbereich "Wissenschaft" wird nach gleichem Grundmuster verfahren. Genannt sei die Textsorte "Forschungsbericht".

Barbara Sandig geht bekanntlich von einer anderen Überlegung aus. Sie sieht in den verschiedenen, durch Kommunikationsbereiche mitgeprägten Grundmustern sogenannte "Mustermischungen" (B. Sandig 1986, 107). Danach weist ein Sportbericht Merkmale der Grundmuster für Bericht und Reportage aus. Der "Erlebnisbericht" ist eine Mischung aus Bericht und Erzählung. Aus unserer kommunikativen Erfahrung wissen wir, daß vom Kommunikationsbereich Erwartungen an die Intention, an die Proposition, an die Sprache bzw. den Stil des Textes, und auch an den Textaufbau ausgehen. Dieser Anspruch ist mit dem Begriff "Mustermischung" nicht ausreichend abgedeckt. Es genügt heute nicht mehr, Kommunikation als Austausch von Informationen zu erklären, die nach konventionellen Mustern verbalisiert und textual organisiert sind. Beschaffenheit und Durchführung einer sprachlichen Handlung werden entscheidend von Erwartungen geprägt, die vom Kommunikationsbereich und dem sozialen Verhältnis der Kommunikationspartner (zusammengenommen von der Kommunikationssituation) ausgehen. Der Einfluß der Kommunikationssituation auf Entscheidungen im sprachlichen Handeln ist gegenüber der Orientierung am überlieferten Textmuster primär. Das erklärt wohl auch, daß die "Mustermischungen" offensichtlich zahlreicher sind als die Muster. Mit dieser Anmerkung möchte ich nicht falsch verstanden werden. Zweifellos gibt es Textmuster, die der Sprecher/Schreiber als Abbild von Textsorten gespeichert hat und die für ihn Orientierungsbasis seiner Textgestaltungsprozesse sind. Es gibt auch weitgehend standardisierte Textmuster, die als Präskriptionen den Textgestaltungsprozeß steuern, z.B. Formulare. Aber in der Mehrzahl der Kommunikationsakte ist der Einfluß der situativen Erwartungen auf das Produkt, also auf den gestalteten Text, bestimmender. Deshalb wird eben der Sportbericht nicht an den Merkmalen eines "Behördenberichts" gemessen werden können. Sportbericht und "Behördenbericht" folgen verschiedenen Grundmustern, die in ihren situativen und deshalb auch in weiteren Merkmalen voneinander abweichen.

Die Medienkommunikation trägt die Handschrift des jeweiligen Informationsträgers, in Deutschland beispielweise "Der Spiegel", "Die Zeit", "Bildzeitung", "ZDF" u.v.a. Ihnen allen ist werbende Information und werbende Unterhaltung für einen zumeist bestimmten Empfängerkreis eigen, sie unterscheiden sich aber darin, wie sie begrifflich thematisieren, Details auswählen und herausarbeiten (vgl. B. Sandig 1986, $278 \mathrm{f}$.), Wirkungen intendieren. Es ist aber nur zum Teil eine sendercharakteristische Realisierung medialer Textsorten, die sich in der Handschrift des jeweiligen Informationsträgers spiegelt; denn im Ergebnis, dem gestalteten Text, spiegeln sich auch die Erwartungen des Lesers/Hörers an die Medienkommunikation. Konkreter: Das Grundmuster zum Medienbericht schließt situative Merkmale der Medienkommunikation ein.

Ich möchte jetzt eine Zusammenfassung meiner Überlegung versuchen, weil sich daraus weitere Fragen ergeben dürften. 

Abbildebene
I theoriebezogene
Kategorie
II Grundmuster
begriffliche Differenzierung
Texttyp (z.B. Bericht)
Textart (z.B. Medienbericht, offizieller Bericht)

III Textsortenmuster Textsorte
Realisierungsebene

$-$

z.B. Sportbericht

z.B. Lebenslauf

Eine Texttypologie arbeitet mit theoriebezogenen Kategorien. Mit Bezug auf sprachliche Handlungstheorien müßte sich folglich eine Typologie ableiten lassen zu Klassen möglicher Intentionen, z.B. informierender, normierender, aktivierender, appellierender, deklarierender Text. Unter wiederum anderem Aspekt können Bezeichnungen für komplex organisierte Handlungen die Basis für eine Typologie abgeben, z.B. Bericht, Erzählung, Erörterung, Beurteilung, Mitteilung, Beschreibung. Aus der Forschungsliteratur sind auch noch andere Vorschläge bekannt.

Texttyp und Textart sind theoretische Konstrukte, die es in der Kommunikation real nicht gibt. Natürlich ist im Alltag und in der Berufssphäre beispielsweise von Berichten und Erzählungen die Rede, aber das sind sprachliche Verallgemeinerungen für eine Klasse konkreter Textvorkommen, eben einer Textsorte. Als eine Textklasse auf der unteren Ebene der begrifflichen Hierarchie ist ihre Bezeichnung ein Hyponym, das heißt ein "Unterbegriff", dem allenfalls noch Varianten zukommen können, z.B. zum "offiziellen Lebenslauf" der tabellarische oder der ausführliche Lebenslauf. Das ist offenbar auch einsichtig und am Bericht noch relativ leicht zu demonstrieren. Aber gerade die Textsortenbeschreibung mit der ihr inhärenten Spezifizierung - gemeint ist die Herausarbeitung artbildender Unterschiede zu anderen Textsorten - bereitet gewisse Schwierigkeiten. Als Beispiel sei nur die Abgrenzung des Lebenslaufs von der Biographie bzw. von der Vita angeführt. Zum anderen folgt die Textsortenbestimmung vorrangig funktionalen Gesichtspunkten, also ihrem Zweck und ihrer Verwendung. Schon deshalb wird es keine erschöpfende Vollkommenheit in der Beschreibung geben können.

Heute ist weitgehend anerkannt, daß die Textsorte als eine stark spezifizierte Textklasse zu beschreiben ist, die auf einen Prototyp zurückgeht. Beschrieben wird der Prototyp. W. Heinemann und D. Viehweger nennen die Textsorte ein "prototypisches Phänomen" (1991, 170). Aus den Alltagserfahrungen im Umgang mit Textsorten sind Strukturmodelle und Merkmalkomplexe entwickelt worden (vgl. die Text-Typologisierung ebenda, $145 \mathrm{ff}$.). Ich ziehe es gleichfalls vor, von einer Merkmalmatrix auszugehen. Typologische Textsortenbestimmungen sind zu allgemein und deshalb wenig praktikabel. Ich verweise auf Typologien, die von Tätigkeitsbereichen ausgehen und ihnen Textsorten zuordnen; zum Beispiel zum Tätigkeitsbereich "Journalistik" der Texttyp "Zeitungstext". und dazu wiederum die Textsorten Nachrichtentext, Bericht, 
Leitartikel, Kommentar, Wirtschaftsteil einer Zeitung. Der tatsächlichen Vielfalt der Realisierungsformen wird nicht entsprochen.

Aus handlungstheoretischer Sicht könnte die Textsorte nach folgenden Merkmalen beschrieben werden:

Sprachhandlung $\rightarrow$

I. situativer Kontext

I.1 Kommunikationsbereich situationsgebundene (z. B. Direktive/Verwalt.)

I.2 Soziale Situation

(z. B. "Bewerber"-Situat.)

II. Intention

(z. B. "werbende" Inform.)

III. Proposition

IV. Kanal

V. Sprache/Stil

VI. Komposition
Indexierung $\rightarrow$

(konventionelle)

Rezipientenerwartung zweckdienlicher

stand

Realisierungsform stimmter Wortklassen Stilzüge, Stiltyp

der Textfunktion ent-

\section{Textsortencharakteristik}

\section{z. B. Lebenslauf}

offiziell

authentisch

tradierte, ggf. besonders dominant informativ

beanspruchte Textfunktion

frei von Redundanz,

Kommunikationsgegen- lückenloser, gewichteter

Lebensbericht

sprachliche Existenzform, schriftlich

Sprachwahl, Gebrauch be- weitgehend konnotationsfreie Lexik, Stilzug: sachbetont chronologisch oder sprechende (in der Regel tradierte) Gliederung/ thematisch geordnet, Themenbereiche chronologisch Anordnung; Themaentfalung

Es ist heute verbreitet üblich, die Textsortenbeschreibung nach textinternen und textexternen Merkmalen vorzunehmen (vgl. H. Bußmann 1990, 781), die der Textsorte eine zweckbestimmte, charakteristische semantische Struktur verleihen. Nur darf diese Struktur nicht als fixe Größe begriffen werden, wie das oft den Anschein hat. Thre prototypische Basis, das Textmuster, wird im Prozeß der Sprachhandlung und unter den konkreten kommunikativen Bedingungen ihrer Realisierung zu einer dynamischen Größe mit einem mehr oder weniger eng gezogenen Realisierungsrahmen.

Würde man beispielsweise nicht vom Texttyp "Bericht", sondern vom Texttyp "Erörterung" ausgehen, ließe sich kaum eine vergleichbare Textsortenstruktur nachweisen. In Schulbüchern ist deshalb nur undifferenziert von der Erörterung die Rede. Gemeinhin verbindet sich mit dem Begriff des Erörterns das Debattieren, das Darlegen des Für und Wider im Prozeß des Klärens oder Lösens von Problemen, das Argumentieren in der Auseinandersetzung um alltägliche und wissenschaftliche Sachverhalte. Wer die Differenzierung sucht, findet als erörternde bzw. argumentierende Textsorten 
allenfalls "Polemik", "Pladoyer", "Rechtfertigung". Hier stellt sich wiederum die Frage, ob man nicht zwischen "Erörterung" und "Argumentation" unterscheiden müsse. Offenbar kennt die Kommunikationspraxis zwar viele Textsorten, aber nicht so viele adäquate Bezeichnungen, so daß die Lücken durch allgemeinere Benennungen geschlossen werden. Offenbar besteht aber auch für eine "Differenzierung auf jeden Fall" weder ein alltägliches noch ein wissenschaftliches Bedürfnis. Eine "Abhandlung" zum Beispiel ist jeder Aufsatz, in dem sich der Verfasser auseinandersetzt, ist jede schriftliche wissenschaftliche Arbeit. Auch ein amtlicher Bericht, der Untersuchungsergebnisse und die Untersuchungsbedingungen darlegt, wird eine Abhandlung genannt.

So undifferenziert wie die allgemeine Benennung ist dann auch die kognitive Existenz entsprechender Textmuster. Ihre sehr flexible Anwendung und folglich auch ihre Abwandlung ist $z w i n g e n d$. Diese Beobachtung hat mich veranlaßt, folgende Typen von Textsorten zu unterscheiden:

I. präskriptiver Typus (das sind weitgehend normierte Textsorten, z.B. Definition, Textsorten auf der Basis von Formularen) (nach W. Eichler, 1992, 341, werden Textsorten, deren Rahmen durch ein Formular vorgegeben wird, gesondert als "formatierte Textsorten" behandelt)

II. usueller Typus (das sind Gebrauchsformen, die nach konventionellen, aber nicht streng verbindlichen Mustern gestaltet werden, z.B. Lebenslauf, Sportbericht)

III. Rahmen-Typus (das sind Anwendungsformen, die nach einem rahmenhaften Grundmuster relativ frei gestaltet sind, z.B. Erörterung, Argumentation)

Der nach einem Muster realisierte Text ist als ein Produkt einer kommunikativen Sprachhandlung immer auch ein Produkt aktueller Entscheidungen. Ein strigentes und in sich geschlossenes System von Textsorten gibt es wohl nicht.

2. Die Textsortenforschung hat bis heute einen Stand erreicht, der uns veranlassen muß, verstärkt über interkulturelle Bezugsmomente nachzudenken. Es geht um die Fragen, ob Textsorten (a) einzelsprachliche, (b) mit Universalien verbundene einzelsprachliche oder (c) übereinzelsprachliche Erscheinungen sind. Die Überlegungen sind nicht neu (ich verweise auf W. Krause 1988, 235 ff.), und es kann auch auf Forschungsergebnisse verwiesen werden. Nach einer Einschätzung von W.-D. Krause $(1988,236)$ "belegen die bisherigen - allerdings nicht sehr zahlreichen Analysen von kommensurablen Texten in verschiedenen Sprachen auf typologischer Ebene, daß Textsorten aufgrund ihrer historisch-gesellschaftlichen Herausbildung und Tradierung in aller Regel bestimmte Prägungen aufweisen, die nur für die entsprechende Kommunikationsgemeinschaft charakteristisch sind. Das betrifft vor allem die makro- und mikrostrukturelle Organisation der Textsorten, aber auch bestimmte sprachlich-formulative Eigenheiten." An anderer Stelle verweist er darauf, daß sich mit Blick auf die internationale Kommunikation, bedingt durch die Internationalisierung der Produktionsprozesse, Tendenzen der "Unifizierung von bestimmten Textsorten" feststellen lassen (ebenda, 237). 
Ein Einstieg in die interkulturelle Textsortenforschung könnte zunächst die vergleichende Betrachtung von Benennungsmotiven sein. Die Motive lassen gegebenenfalls erste, aber keinesfalls sichere Rückschlüsse auf Wesen und Funktion der jeweiligen Textsorte zu. Beispielsweise geht die im deutschsprachigen Raum übliche Bezeichnung "Protokoll" für die wörtliche oder dem Sinn nach erfolgte Mitschrift zu Verlauf und/oder Ergebnissen einer Beratung, Sitzung, Versammlung, Vereinbarung auf mlat. $>$ protocollum< zurück. Das ist ursprünglich ein Blatt mit chronologischen Angaben, das einer amtlichen Papyrusrolle vorgeleimt war. Geblieben in der nur etymologisch erschließbaren semantischen Struktur ist der amtliche Charakter des Protokolls und die chronologische Anordnung der niedergeschriebenen Informationen. Eine adäquate Bezeichnung in der slowenischen Sprache ist "zapisnik", was wörtlich "Sitzungsniederschrift" bedeutet. Es ist eine Ableitung zu "zapisik" bzw. "zapis" mit der aktuellen Bedeutung "Niederschrift". Das Benennungsmotiv ist im Unterschied zur deutschen Bezeichnung morphologisch durchsichtig, und es ist mit der Beschränkung auf "Niederschrift zu einer Sitzung" weniger extensional. Strukturelle bzw. formulative Unterschiede zwischen slowenischen und deutschen Protokollen bestehen nicht. Traditionell üblich ist der Protokollkopf und die sachbetonte Form der Niederschrift.

Adäquat zu deutsch "Lebenslauf" wird im Slowenischen "življenjepis" verwendet. "Pis" entspricht dem deutschen Wortstamm -schreib- (vgl. Schreiber - pisar). Das auch selbständig gebräuchliche Substantiv "življenje" ist in der semantischen Struktur identisch mit dem deutschen Lexem "Leben". Während die deutsche Textsortenbezeichnung "Lebenslauf" das Prozessuale heraushebt (Verlauf, Gang des Lebens; slow. "Lebensgang" = potek življenja), ist die slowenische Textsortenbezeichnung durch "Beschreibung, Niederschrift des Lebens" motiviert. Im Verständnis der slowenischen Sprachgemeinschaft ist "življenjepis" auf die schriftliche Form festgelegt. Untypisch, aber nicht ungewöhnlich ist die Bildung eines Kompositums, denn sie ist für slawische Sprachsysteme nicht wesenhaft.

Neben den Unterschieden in den Benennungsmotiven fallen auch unterschiedliche sprachlich-formulative Eigenheiten auf. Deutsche ausführliche Lebensläufe beginnen wenn auch nicht zwingend - mit der Präposition "am" und dem Geburtstag: "Am 28.09.1964 wurde ich in X geboren." Der Personenname als Apposition zum Pronomen "ich" erscheint in der Regel nicht mehr in dieser grammatischen Form. Insofern hat sich der Prototyp mit der Durchsetzung freierer Stilentscheidungen verändert. Der Liberalisierung bei der Anwendung von Gebrauchsmustern stehen aber Verbindlichkeiten entgegen, weil Textsorten Funktionen in der Kommunikation haben. Für den ausführlichen Lebenslauf eines Bewerbers werden angemessene Offizialität, sprachliche Neutralität (durch konnotationsfreie Lexik), Übersichtlichkeit, gekonnte Formulierung und eine der speziellen Funktion angemessene Auswahl von Lebensdaten erwartet. Zur Übersichtlichkeit gehört, daß die Textsortenbezeichnung und der Name der Person, um die es geht, in der Texteröffnung hervorgehoben sind. Die handschriftliche Fassung des Lebenslaufs kann verlangt werden. Die Erwartungen sind durch den Kommunika- 
tionsbereich, den Kommunikationspartner und die Konvention gesetzt. Wir wissen, daß die Erfüllung der formalen Ansprüche Entscheidungen beeinflussen kann.

Für die vergleichbare slowenische Textsorte wird die Standarderöffnung gebraucht: "Podpisana Vida S..., roj. 28.09.1964 v X ..." (Unterzeichnete Vida S..., geb. am 28.09.1964 in X ...). Abweichungen davon bilden eine Ausnahme. Meine Studenten empfinden, daß der Grad des Sachlich-Offiziellen slowenischer Lebensläufe höher anzusetzen ist als im Deutschen. Die für den deutschen Lebenslauf herausgestellten Erwartungen gelten auch für den slowenischen.

Zweifellos wird bei diesem recht oberflächlichen Vergleich der Mangel an formalen und semantischen Analysen in der interkulturell orientierten Textsortenforschung spürbar, und, indem wir das konventionelle Moment betonen, wird uns auch der Mangel an historischen Kenntnissen bewußt. Beispielsweise ist "živiljenjepis" zweifelsfrei eine systemfremde künstliche Bildung vermutlich im 19. Jahrhundert. Sie hat ihre historischen Ursachen, die für die Erklärung der Textsorten-Stilform wesentlich sein könnten.

Die semantische Analyse der Textsortenbenennungen hat mehr einen methodischen als einen wissenschaftlichen Wert, weil sie keinen sicheren Hinweis über die Mikro- und Makrostruktur der Textsorten geben kann, auch nicht über ihre speziellen Formalia und ihre tatsächlichen Funktionen in der Kommunikation. Aber sie kann ein Einstieg in die Untersuchung sein.

Der Vergleich von Sprachen auf Textebene hat einen textwissenschaftlichen wie auch praktischen Wert. Er liefert Aufschlüsse über die konstitutiven Eigenschaften von Texten in kommunikativen Zusammenhängen. Davon profitiert nicht nur die Textlinguistik. Auch die Ethologie (Lehre von den Sitten und Gebräuchen eines Volkes), die Soziologie und die Universalienforschung könnten ein Interesse daran haben. Außer Frage steht der Wert für die Translation. Ich meine sogar, daß die Überbetonung des Einzelsprachlichen wie auch die Überbewertung einzelsprachlicher Analyseergebnisse die Textsortenforschung nicht voranbringt, weil sie den konventionellen Rahmen für sprachlich-kommunikatives Handeln zu eng definiert. Über meine Ansicht mag man streiten, gleichwohl, ich akzeptiere auch das Praxis-Argument, daß jedes interdisziplinäre wie auch interkulturelle Herangehen relative Gewißheiten und eine eingehende Klärung auf disziplinärer und kultureller Ebene erfordert. Aber eben diese kulturelle Ebene werden wir in der Sprachverwendungs- und Textforschung nicht vor allem beibehalten dürfen. In einer Zeit, in der interkulturelle Kontakte über die Massenmedien, über die Internationalisierung der Wirtschaft, über den Tourismus intensiviert werden, sichert die Sprachbeherrschung allein keine erfolgreiche Kommunikation. Das Wissen um Sprachverwendungsregeln und kulturell geprägte Eigenheiten der Textbildung ist ebenso vonnöten. Die Sprache jedes Volkes ist ein Teil seiner Kultur; aber auch seine Geschichte, seine Religion, das erwartbare Sozialverhalten in der Kommunikation, überkommene Regularitäten im Zusammenleben, das sind wesentliche Elemente der Volkskultur, und es wird zu recht erwartet, daß man sie respektiert. Aus ihnen sind Verhaltensformen in der Kommunikation erwachsen, aber auch ethnisch 
geprägte Eigenheiten in den Textsortennormen, die unter dem Oberbegriff der "interkulturellen Verschiedenheiten" zusammengefaßt werden (vgl. auch E. Oksaar 1988, 5).

Mit meinem Beitrag möchte ich mehr Forschungsbedarf für eine interkulturell gerichtete Textsortenforschung anmelden. Ich möchte den interkulturellen Verschiedenheiten aber auch kein Übergewicht beimessen, denn das Gemeinsame, das Übereinzelsprachliche zu erkennen ist ebenso wichtig. Es fällt vor allem dort ins Gewicht, wo sich verschiedene Kulturen eng berühren.

\section{Literatur}

- Bußmann, Hadumod: Lexikon der Sprachwissenschaft. Stuttgart 1990

- Eichler, Wolfgang: Zum Zusammenhang zwischen gestalteten Aufsatzarten und pragmatischen Textsorten. In: Deutschunterricht. Heft 7/8, Berlin 1992, Seiten 338 352

- Gülich, Elisabeth: Textsorten im Kommunikationsprozeß. - Kommunikationstypologie. Handlungsmuster, Textsorten, Situationstypen. Jahrbuch 1985 des Instituts für deutsche Sprache. Düsseldorf 1986, Seiten $15-46$

- Gülich, Elisabeth/ Raible, Wolfgang (als Hrsg.): Textsorten, Differenzierungskriterien aus linguistischer Sicht. Frankfurt/Main 1972

- Heinemannn, Wolfgang: Textsorten/Textmuster - ein Problemaufriß. In: Textsorten und Textmuster in der Sprech- und Schriftkommunikation. Leipzig 1990, Seiten 8 - 16 - Heinemann, Wolfgang/ Viehweger, Dieter: Textlinguistik. - Eine Einführung. Tübingen 1991

- Krause, Wolf-Dieter: Zur Präzisierung eines sprachwissenschaftlichen Textsortenbegriffs. In: Wiss. Ztschr. der PH Potsdam, Heft 5, Potsdam 1988, Seiten $233-240$

- Lenk, Hartmut E.H.:Praktische Textsortenlehre. Ein Lehr- und Handbuch der professionellen Textgestaltung. Helsinki 1993

- Oksaar, Els: Kulturemtheorie. - Ein Beitrag zur Sprachverwendungsforschung. Hamburg 1988

- Sandig, Barbara: Zur Differenzierung gebrauchssprachlicher Textsorten im Deutschen. In: E. Gülich/W. Raible (als Hrsg.): Textsorten. Differenzierungskriterien aus linguistischer Sicht. Frankfurt/Main 1972, Seiten 113 - 124

- Sandig, Barbara: Stilistik der deutschen Sprache. Berlin, New York 1986

\section{Zusammenfassung}

Der Begriff der Textsorte ist an deutschen Schulen bereits als Schulterminus geläufig, dennoch ist er noch immer umstrittener Gegenstand in der wissenschaftlichen 
Diskussion. Relativ gesichert ist die Erkenntnis, daß Textsorten Klassen realer Textvorkommen sind, für die auf der Abbildebene teils überlieferte, teils aus der Erfahrung (bzw. der beruflichen Praxis) Muster angenommen werden können, nach denen z.B. Protokolle, Lebensläufe, Geschäftsbriefe geschrieben werden. Die Text- bzw- Kommunikationslinguistik sieht sich vor die Aufgabe einer wissenschaftlichen Beschreibung gestellt, was im Alltagsbewußtsein bereits recht fest, aber doch oft unbestimmt verankert ist.

Der Beitrag stellt verschiedene Auffassungen zur Textsortenproblematik heraus, er verweist aber auch auf Fortschritte, die die wissenschaftliche Diskussion bisher gebracht hat. Dennoch muß man die Tatsache anerkennen, daß es ein strigentes und in sich geschlossenes System von Textsorten wohl nicht gibt. Textsorten sind nichts Statisches, denn sie unterliegen Entwicklungen in den kommunikativen Erwartungen, sie unterliegen aber auch den verschiedenen Anforderungen der Kommunikationsbereiche (man denke nur an die verschiedenen Funktionen, die offizielle Berichte in Verwaltung und Wissenschaft haben gegenüber Ansprüchen an Medienberichte). Ebenso kann auch der Textstil nicht gänzlich vorgeschrieben werden. Ihm ist immer auch Individuelles eigen.

Der Beitrag nimmt die wissenschaftliche Beschreibung von Textsorten von ihrer Entstehung im Textgestaltungsprozeß (die Sprachhandlung) her auf und leitet daraus Charakteristika $a b$, die bezogen sind auf die Proposition, die Intention, den Kommunikationskanal, den Textaufbau und die sprachliche Realisierung des Textes.

Ein deutlich weiterweisender Aspekt ist die Textsorte in der interkulturellen Kommunikation. Ansätze dafür werden aufgezeigt, z.B. die Realisierung der Textsorte "Lebenslauf" im Deutschen und im Slowenischen. Der Verfasser meldet Forschungsbedarf für eine interkulturell gerichtete Textsortenforschung an.

\section{Povzetek}

\section{BESEDILNE VRSTE V MEDKULTURNI KOMUNIKACIII - ORIS PROBLEMA}

Pojem "besedilna vrsta" je v nemških šolah ze uveljavljen termin, vendar pa je v znanosti še vedno sporen. $S$ precejšnjo gotovostjo lahko trdimo, da so besedilne vrste skupine konkretnih besedil; za njihovo abstraktno ponazoritev lahko služijo vzorci, ki so delno prevzeti, delno pa pridobljeni s pomočjo izkušenj (oziroma poklicne prakse). Ti vzorci služijo kot predloga pri pisanju denimo zapisnikov, življenjepisov in poslovnih pisem. Naloga besediloslovja oziroma sporočanja je znanstveno opisati to, kar je v vsakodnevni praksi že trdno zasidrano, česar pa pogosto ni moč točno določiti.

V prispevku predstavljam različna pojmovanja problema besedilne vrste, obenem pa opozarjam na napredek, ki ga je znanost dosegla na tem področju. Vendarle pa je treba priznati, da zaprt sistem besedilnih vrst takorekoč ne obstaja. Besedilnih vrst ne gre pojmovati kot nekaj statičnega. Nanje namreč vplivajo razvojne tendence $\mathrm{v}$ komunikativnih pričakovanjih, pa tudi različna komunikativna področja (pomislimo le na različne funkcije uradnih poročil na področju uprave in znanosti v primerjavi z medijskimi poročili). Prav tako ni mogoče povsem predpisati besedilnega stila. $\mathrm{V}$ njem se vedno odraža tudi stil posameznika. 
Prispevek predstavlja znanstveni opis besedilnih vrst in sicer od zasnove v procesu oblikovanja besedila ter od tod izpelje značilnosti glede na propozicijo, namen, prenosnik, zgradbo besedila in njegovo jezikovno realizacijo.

V okviru prispevka je jasno začrtan vidik besedilne vrste v medkulturni komunikaciji, pri čemer avtor poda nekaj iztočnic za tovrstne raziskave: denimo realizacija besedilne vrste "življenjepis" v nemščini in slovenščni, obenem pa opozori na potrebo po raziskavah na medkulturni ravni. 